\title{
Fatty Acid Methyl Ester Profile Analysis of In-Vitro Grown Accessions of Plumbago zeylanica
}

\section{Arpita Roy, Neeru Thakran and Navneeta Bharadvaja*}

Department of Biotechnology, Plant Biotechnology Laboratory, Delhi Technological University, New Delhi-110042, India

*Corresponding author: Bharadvaja N, Department of Biotechnology, Plant Biotechnology Laboratory, Delhi Technological University, New Delhi-110042, India, Tel: 011 2787 1018; E-mail: navneetab@dce.ac.in

Received: April 10, 2018; Accepted: April 17, 2018; Published: April 23, 2018

Copyright: () 2018 Roy A, et al. This is an open-access article distributed under the terms of the Creative Commons Attribution License, which permits unrestricted use, distribution, and reproduction in any medium, provided the original author and source are credited.

\begin{abstract}
Plumbago zeylanica is a pharmaceutically important plant which belongs to Plumbaginaceae family. It possesses wide range of pharmacological activities which includes antibacterial central nervous system stimulatory activity, anti-fungal, anti-inflammatory, anti-hyperglycemic, anti-cancer and anti-atherosclerotic activity. Fatty acid methyl ester analysis reveals the presence of various fatty acids in the plant. The present study deals with Fatty acid methyl ester profile of five different accession of Plumbago zeylanica. Results reveal that these accessions are rich in Octadecadienoic acid (8-22\%), Octadecatrienoic acid (7-24\%) and Pentadecanoic acid (11-22\%) fatty acids. Accession number 421418 is contains the highest amount of Pentadecanoic acid and 9,12,15 Octadecatrienoic acid. Accession number 439212 contains highest amount of Hexadecanoic acid. Highest amount of 9,12 Octadecadienoic acid was present in accession number 524441.
\end{abstract}

Keywords: Plumbago zeylanica; Accessions; Fatty acid methyl ester; MS medium

\section{Introduction}

Plumbago zeylanica is an important medicinal plant which belongs to the Plumbaginaceae family. It is commonly known as 'Chitra' and grows in the Bengal and Southern India [1]. It's a perennial herb and widely used in the treatment of diseases such as diarrhoea, dyspepsia, skin diseases, piles, rheumatism and leprosy [2]. It contains a variety of secondary metabolites like glycosides, steroids, saponins, flavonoids, alkaloids, tri-terpenoides, tannins, coumarins, phenolic compounds, carbohydrates, fixed oils, fats, proteins and napthoquinones [3,4]. It possesses anti-bacterial, anti-fungal [5] anti-cancer [6], antiplasmodial, anti-tumour, hepatoprotective, central nervous system stimulatory activity, anti-inflammatory [7], anti-hyperglycemic, antiatherosclerotic activity [8]. An accession means collection of plant material of single species from a specific geographical location. Each accession is an attempt to capture the diversity present in a given plant population. Accession number is given a unique identifier, and it is used to maintain associated information in the database. It exhibits significant variations in morphological parameters like growth of leaf, flowering, stomatal frequency, etc. The purpose of using different accession was to choose the best accession for phytocompounds production [9]. Fatty acids are identified as active ingredients in herbal medicine. FAME analysis provides an extensive knowledge of volatile compounds present in the plants. Fatty acid profiling known as FAME determines presence of fatty acids in the sample. This process hydrolyzes fatty acids from triacylglycerols, phospholipids, sterols, and various other lipid structures and then adds a methyl group to carboxyl group of fatty acids which forms a methyl ester that acts as a label for the Gas Chromatography, which then reads and identifies those fatty acids. The system was termed FAME analysis since it utilizes fatty acid methyl esters. Resulting composition profile provides the critical assistance in valuable oil present in the sample. Unsaturated fatty acids which includes monounsaturated fatty acids (MUFA) and polyunsaturated fatty acids (PUFA) are health-promoting and have significant metabolic and cardiovascular benefits [10]. Fatty acids play important role in the human diet, it is estimated that human brain contains nearly $60 \%$ fat [11]. Usually essential fatty acids are found in plants and they play vital role in brain functions [12]. They also regulate lymphocyte metabolism and proliferation induces $\mathrm{T}$ cell death and enhance auto-immunity [13]. Fatty acids also possess anti-cancer activity [14]. There is no reported study about the FAME analysis in the leaves of $P$. zeylanica. Thus, an attempt has been made to study the FAME analysis in this plant.

\section{Materials and Methods}

\section{Plant material}

Five different accessions of $P$. zeylanica i.e., 398891, 524441, 421418, 439212, and 539866 were collected from NBPGR, New Delhi, India. They were maintained in MS basal media in the Plant Biotechnology Laboratory, Delhi Technological University.

\section{Fatty acid methyl ester (FAME) analysis of accessions}

For FAME analysis, $100 \mathrm{mg}$ leaves of each accession of Plumbago zeylanica were slightly crushed in mortar pestle and then transferred to a screw-cap glass tubes. $1 \mathrm{ml}$ of $2 \%$ methanolic $\mathrm{HCl}$ was added to the crushed leaves and samples were then incubated at $90^{\circ} \mathrm{C}$ for an hour. After that $0.9 \%$ of $\mathrm{NaCl}$ solution in water was added followed by $2 \mathrm{ml}$ of hexane and mixed. Samples were then centrifuged for phase separation at $2000 \mathrm{rpm}$ for 2 minutes. After centrifugation, the upper (hexane) layer of the sample was transferred into a fresh glass tube and dried under nitrogen flow. Dried samples were then diluted with the $100 \mu \mathrm{l}$ of hexane. One by one $1 \mu \mathrm{l}$ sample were then injected into GCMS analysis. 
Citation: $\quad$ Roy A, Thakran N, Bharadvaja N (2018) Fatty Acid Methyl Ester Profile Analysis of In-Vitro Grown Accessions of Plumbago zeylanica. Nat Prod Chem Res 6: 320. doi:10.4172/2329-6836.1000320

Page 2 of 5

\section{Results and Discussion}

\section{Fatty acid methyl ester (FAME) production and analysis}

Fatty acid as well as their relative percentage composition obtained from the GC-MS analysis of the n-hexane extracts of ive different accessions of Plumbago zeylanica (Table 1 and Figures 1a-1e). The plant sample contain various fatty acids, among which Octadecadienoic acid (8-22\%), Octadecatrienoic acid (7-24\%) and Pentadecanoic acid (11-22\%) being present in maximum amounts as per the chromatograms obtained. Tetradecanoic acid (0-2\%), Hexadecanoic acid (1-2\%) and Octadecanoic acid (1-7\%) were also obtained but comparatively low amount in all the ive accessions of $P$. zeylanica. Accession number 524441 contains maximum number of fatty acids compared to the other accessions. Unsaturated fatty acids, Octadecadienoic and Octadecatrienoic acid are the most important essential fatty acids as our body cannot synthesize these fatty acids.
Accession no. 539866 contain highest percent of Octadecadienoic acid (approximately 23\%) followed by 524441 (18.45\%), 398891 (14.57\%), $421418(12.38 \%)$ and $439212(8.87 \%)$. When we consider the presence of Octadecatrienoic acids, it is recorded that accession no. 421418 contain the highest percent approx. 24\% followed by 43921 (9.05\%), 524441 (7.85\%), 539866 (7.53\%) and 398891 (7.004\%). Linoleic acid (Octadecadienoic acid) is essential for maintenance of growth and shown to be potent cycloxygenase-2 (COX-2) catalysed prostaglandin biosynthesis inhibitors. Palmitic acid (Hexadecanoic acid) reduces the risk of cardiovascular disease. Stearic acid used in baked food items. Only three accessions of $P$. zeylanica contain approximately 2 percent of Hexadecanoic acid (stearic acid) (Table 1). Thus it can be concluded that this plant is a good source of fatty acids and can be used in cholesterol-lowering diets. Similar study has been conducted by Roy et al. [15] in case of Centella asiatica where they have reported the presence of Pentadecanoic, Hexadecanoic and Octadecanoic acid, Octadecadienoic and Octadecatrienoic.

\begin{tabular}{|l|l|l|l|l|l|}
\hline \multirow{2}{*}{ Fatty Acid } & \multicolumn{4}{l|}{ Relative Percentage of Fatty acid in different Accessions } \\
\cline { 2 - 7 } & $\mathbf{4 2 1 4 1 8}$ & $\mathbf{3 9 8 8 9 1}$ & $\mathbf{5 3 9 8 6 6}$ & $\mathbf{4 3 9 2 1 2}$ & $\mathbf{5 2 4 4 4 1}$ \\
\hline Tetradecanoic acid & 0 & 1.16 & 0 & 0 & 0.47 \\
\hline Pentadecanoic acid & 22.33 & 11.62 & 15.69 & 17.66 & 15.27 \\
\hline Hexadecanoic acid & 0 & 0 & 1.45 & 2.18 & 1.12 \\
\hline Octadecanoic acid & 1.57 & 4.17 & 5.87 & 4.16 & 6.65 \\
\hline 9,12 Octadecadienoic acid & 12.38 & 14.57 & 22.46 & 8.87 & 18.45 \\
\hline $9,12,15$ Octadecatrienoic acid & 23.86 & 7.004 & 7.53 & 9.05 & 7.85 \\
\hline Total & 60.14 & 38.52 & 53 & 41.92 & 49.81 \\
\hline
\end{tabular}

Table 1: Percentage of methylated fatty acids of five different accession of Plumbago zeylanica.

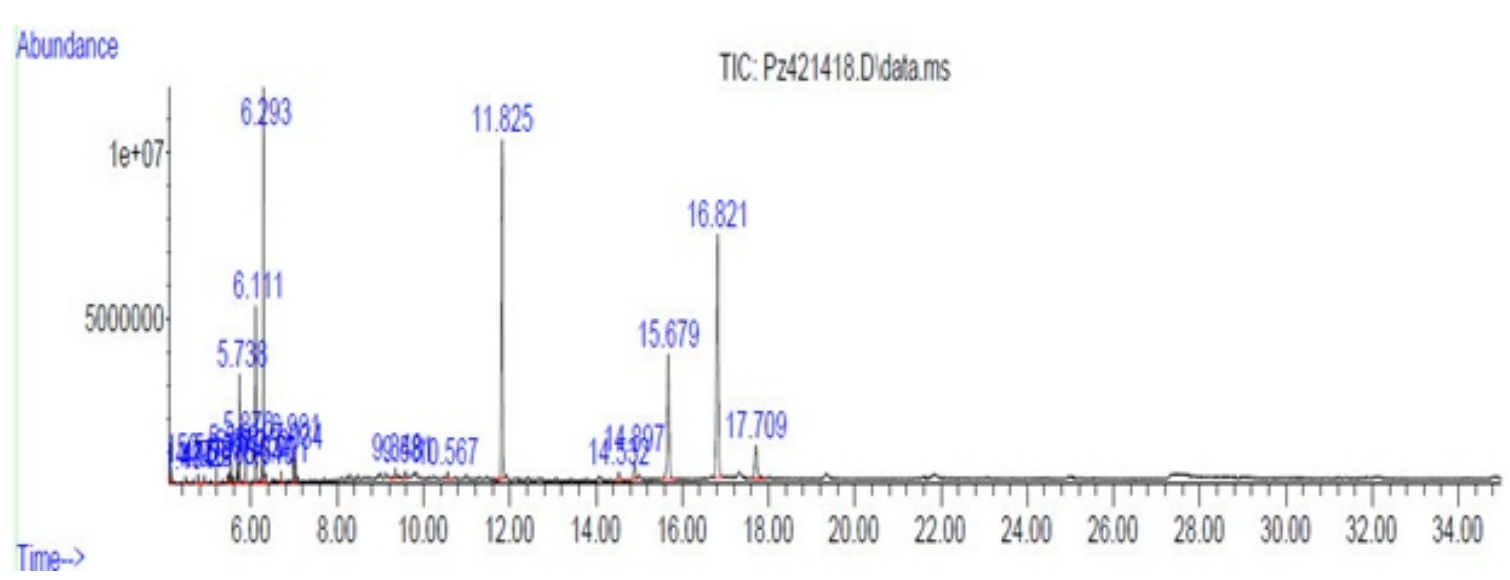

Figure 1a: Chromatogram of Plumbago zeylanica accession no. 421418. 
Citation: $\quad$ Roy A, Thakran N, Bharadvaja N (2018) Fatty Acid Methyl Ester Profile Analysis of In-Vitro Grown Accessions of Plumbago zeylanica. Nat Prod Chem Res 6: 320. doi:10.4172/2329-6836.1000320

Page 3 of 5

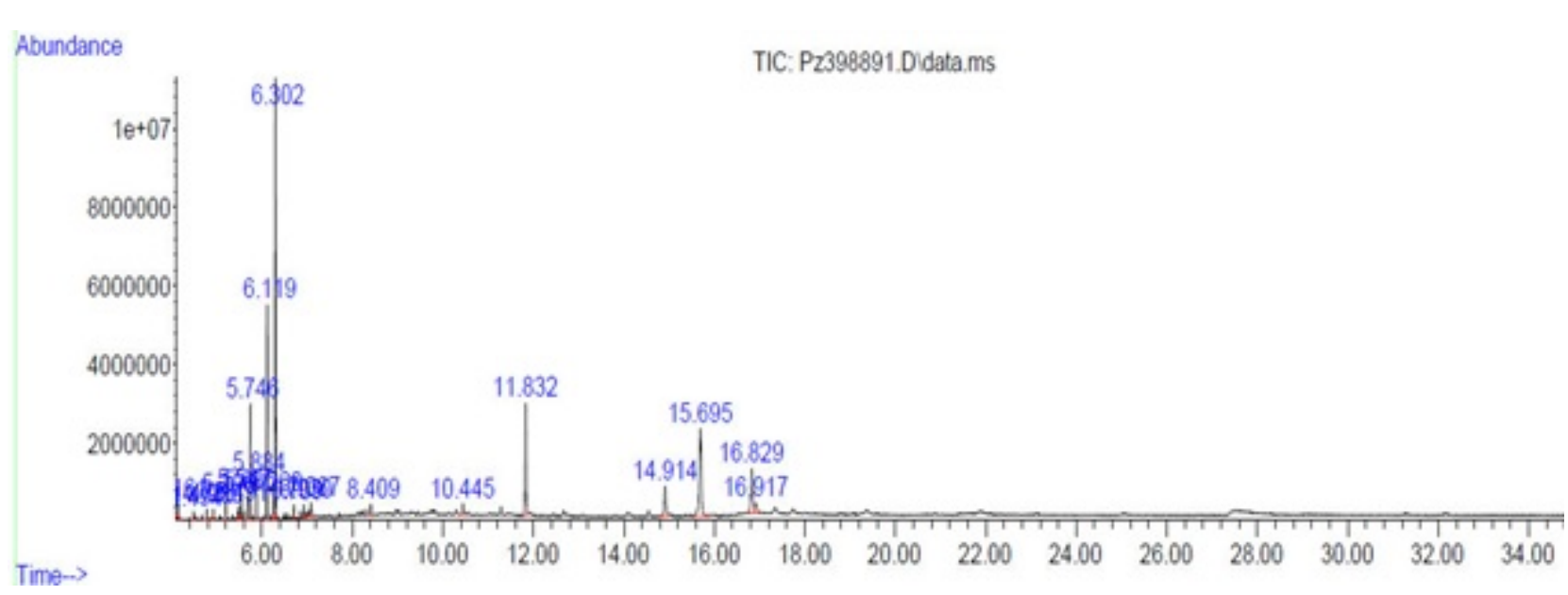

Figure 1b: Chromatogram of Plumbago zeylanica accession no. 398891.

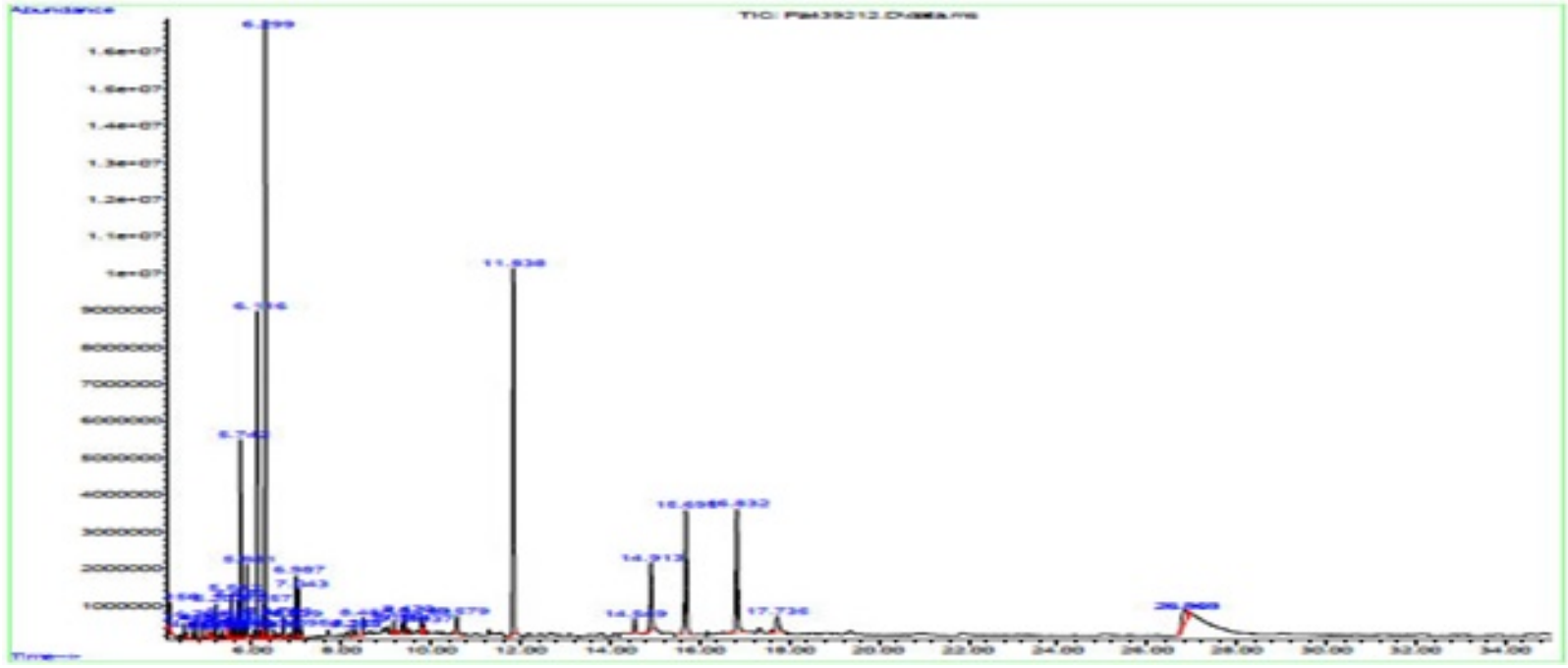

Figure 1c: Chromatogram of Plumbago zeylanica accession no. 539866. 
Citation: $\quad$ Roy A, Thakran N, Bharadvaja N (2018) Fatty Acid Methyl Ester Profile Analysis of In-Vitro Grown Accessions of Plumbago zeylanica.

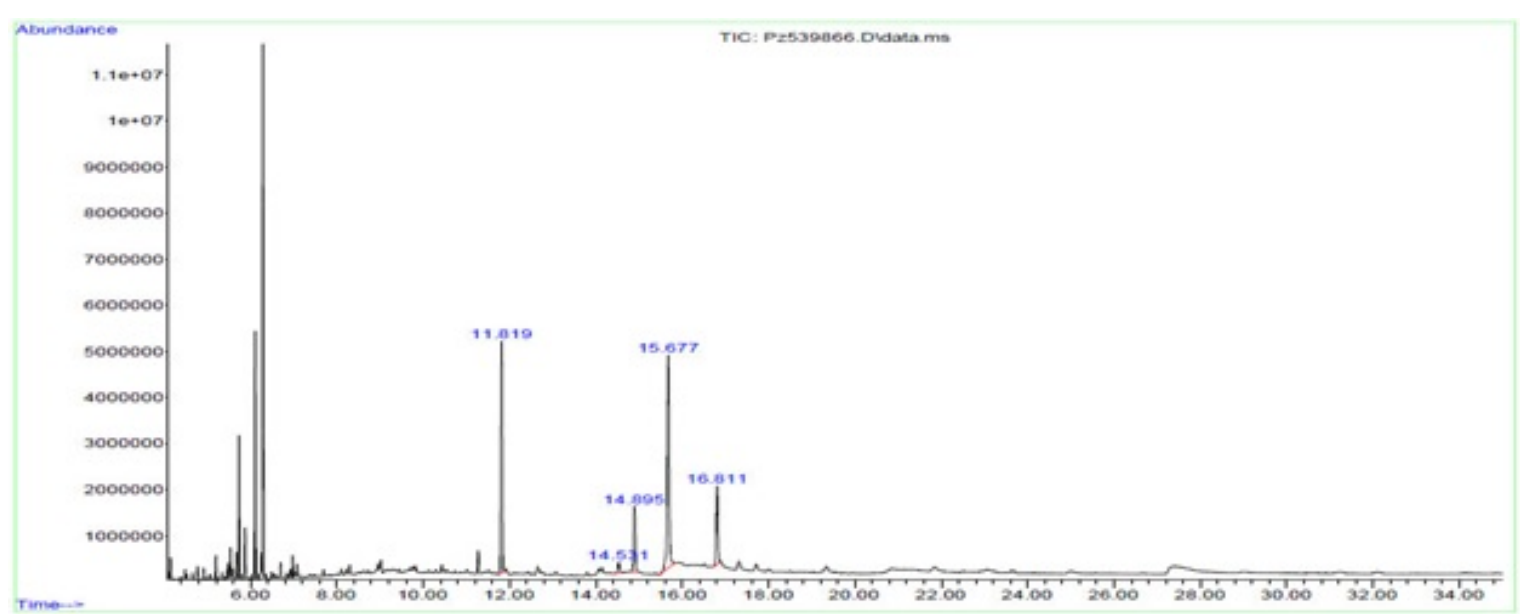

Figure 1d: Chromatogram of Plumbago zeylanica accession no. 439212.

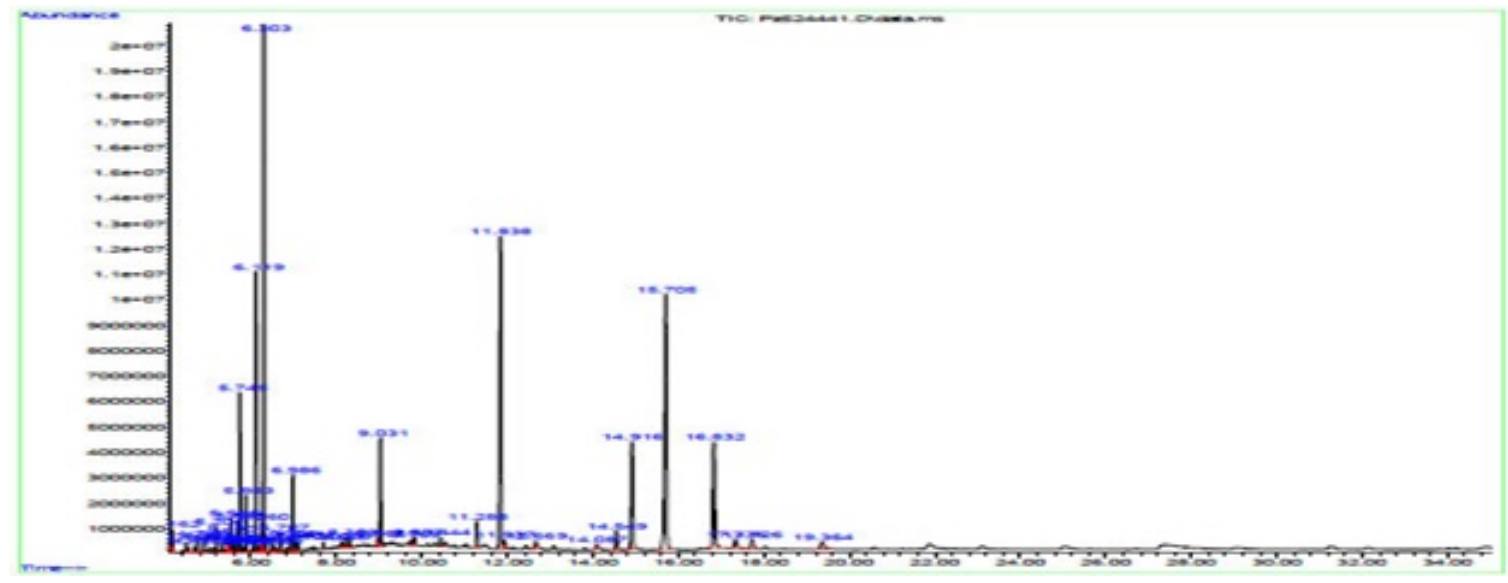

Figure 1e: Chromatogram of Plumbago zeylanica accession no. 524441.

\section{Conclusion}

FAME analysis respectively showed that this plant is rich in Octadecadienoic acid (8-22\%), Octadecatrienoic acid (7-24\%), Pentadecanoic acid (11-22\%) fatty acids. Pentadecanoic acid, Hexadecanoic acid, Octadecanoic acid are saturated fatty acids while other are unsaturated fatty acids. Accession number 421418 showed highest amount of Pentadecanoic acid and 9,12,15 Octadecatrienoic acid.

\section{Acknowledgements}

Our sincere thanks goes to Department of Biotechnology, Delhi Technological University for providing all the facilities.

\section{References}

1. Sharma A, Singh N (2015) A multifarious potent herb: Plumbago zeylanica-a mini review. Intern J Recent Sci Res 6: 4825-4829.
2. Roy A, Bharadvaja N (2017) Establishment of the Shoot and Callus Culture of an Important Medicinal Plant Plumbago zeylanica. Adv Plants Agric Res 7: 274

3. Ming Y, Wang J, Yang J, Liu W (2011) Chemical constituents of Plumbago zeylanica. Adv Mat Res 308: 1662-1664.

4. Roy A, Bharadvaja N (2017) Medicinal Plants in the Management of Cancer: A Review. Int J Complement Alt Med 9: 291.

5. Uma Devi P, Soloman FE, Sharda AC (1999) Plumbagin, a plant naphthoquinone with antitumor and radiomodifying properties. Pharmaceut Biol 37: 231-236.

6. Roy A, Bharadvaja N (2017) Silver Nanoparticles Synthesis from a Pharmaceutically Important Medicinal Plant Plumbago Zeylanica. MOJ Bioequiv Availab 3: 46

7. Arunachalam KD, Velmurugan P, Raja RB (2010) Anti-inflammatory and cytotoxic effects of extract from plumbago zeylanica. African J Microbio Res 4: 1239-1245.

8. Kumar R, Kumar S, Patra A, Jayalakshmi S (2009) Hepatoprotective activity of aerial parts of Plumbago zeylanica linn against carbon tetrachloride-induced hepatotoxicity in rats. Internl J Pharm Pharmace Sci 1: 171-175. 
Citation: $\quad$ Roy A, Thakran N, Bharadvaja N (2018) Fatty Acid Methyl Ester Profile Analysis of In-Vitro Grown Accessions of Plumbago zeylanica. Nat Prod Chem Res 6: 320. doi:10.4172/2329-6836.1000320

Page 5 of 5

9. Roy A, Kundu K, Saxena G, Kumar L, Bharadvaja N (2016) Effect of Different Media and Growth Hormones on Shoot Multiplication of In Vitro Grown Centella asiatica Accessions. Adv Tech Biol Med 4: 172.

10. Gillingham LG, Harris-Janz S, Jones PJ (2011) Dietary monounsaturated fatty acids are protective against metabolic syndrome and cardiovascular disease risk factors. Lipids 46: 209-228.

11. Chang CY, Ke DS, Chen JY (2009) Essential fatty acids and human brain. Acta Neurol Taiwan 18: 231-241.

12. Hadley KB, Ryan AS, Forsyth S, Gautier S, Salem N (2016) The Essentiality of Arachidonic Acid in Infant Development. Nutri 8: 216.
13. Kaur N, Chugh V, Gupta AK (2014) Essential fatty acids as functional components of foods-A Review. J Food Sci Technol 51: 2289-2303.

14. Simon JA, Chen YH, Bent S (2009) The relation of alpha-linolenic acid to the risk of prostate cancer: A Systematic review and meta-analysis. Am J Clin Nutr 89: 1558-1564.

15. Roy A, Kundu K, Saxena G, Bharadvaja N (2017) Estimation of Asiaticoside by Using RP-HPLC and FAME Analysis of Medicinally Important Plant Centella asiatica. J Plant Biochem Physiol 5: 198. 